\title{
Muse Headband: Potential Communication Tool for Locked-in People
}

\author{
Kaihao $\operatorname{Tian}^{1}$ \\ ${ }^{1}$ Cate School, California, USA \\ Correspondence: Kaihao Tian, Cate School, California, USA. E-mail: ktian0222@qq.com
}

Received: November 10, 2017

Accepted: November 29, 2017 Online Published: February 27, 2018

doi:10.5539/mer.v8n1p16

URL: https://doi.org/10.5539/mer.v8n1p16

\begin{abstract}
As an affordable brain wave sensing device, Muse Headband has a strong potential use of being a basic communication tool for people with locked-in syndrome. With the assistance of other external devices, such as EEG and computers, it is possible to create an affordable device that displays these patients' emotions with simple and comprehensible images or graphs so that they can be understood by those who take care of them. My paper introduces Unlock, an in-progress design project based on Muse Headband. The whole program is designed as an approach to help the communication of locked-in people, and consists of both hardware and software.
\end{abstract}

Keywords: Muse Headband, communication assistance, locked-in people, design project

\section{Introduction}

\subsection{Research Goal}

The main purpose of this research is to explore Muse Headband's potential use as a basic communication tool for people with locked-in syndrome. Locked-in syndrome is a condition in which a person is conscious but not able to move or speak due to complete paralysis of nearly all voluntary muscles in the body except for vertical eye movements and blinking. With the assistance of other external devices, such as EEG and computers, it is possible to create an affordable device that displays these patients' emotions with simple and comprehensible images or graphs so that they can be understood by those who take care of them.

\subsection{Motivation}

The main inspiration for my research paper comes from my grandfather, who passed away a few years ago. My grandfather had Parkinson's Disease, a long-term degenerative disorder that affects the basal ganglia of the nervous system. At first, he suffered from symptoms like shaking and limping. In the late stages of the disease, however, he was incapable of moving most of his body at all. The doctors were obliged to remove my grandfather's trachea, since his lung had become infected. Along with his trachea, he lost his ability to speak, which was the only remaining method for him to communicate directly with all my other family members-my dad, my mom, my grandmother, and I. Despite being full conscious, he could only communicate with us through blinking, since his eyes were the only body parts he was able to move. It was a hard time. My grandfather was finally relieved of all the pain brought on by his disease when he passed away a few months later.

I often miss my grandfather. I saw his pain clearly as a child, and it left a deep impression on me. When we asked him whether he was happy or not, he blinked again and again and again, communicating that he was happy for all of us to be with him. For a long time, I couldn't help but wonder how different it would have been if he could have expressed his feelings in other ways and without struggle, just as a small compensation for the countless abilities he lost. There are so many patients like my grandfather. Some of them cannot even move their eyes so they are entirely mentally isolated from the rest of the world and from their loved-ones. My motivation for this project is simple: I want to find a way to help these locked-in people communicate with others using brain wave sensing technology, and I want to design a device with a straightforward interface to display the feelings of locked-in people.

\section{Background}

\subsection{Tool: Muse Headband}

Muse Headband, an affordable brain wave sensing device, has been popular among its users. With sensors at different positions, the headband is capable of detecting and classifying 5 different waves in our brain: Delta, Theta, Alpha, Beta, and Gamma. Delta waves are most present during deep sleep. Theta waves are associated with drowsiness, light sleep, and visualization. Alpha waves occur during wakeful relaxation. Beta waves occur when one is alert, actively 
thinking or problem solving. Gamma waves occur when one is involved in higher mental activity and consolidation of information.

\section{Brain Waves Graph}

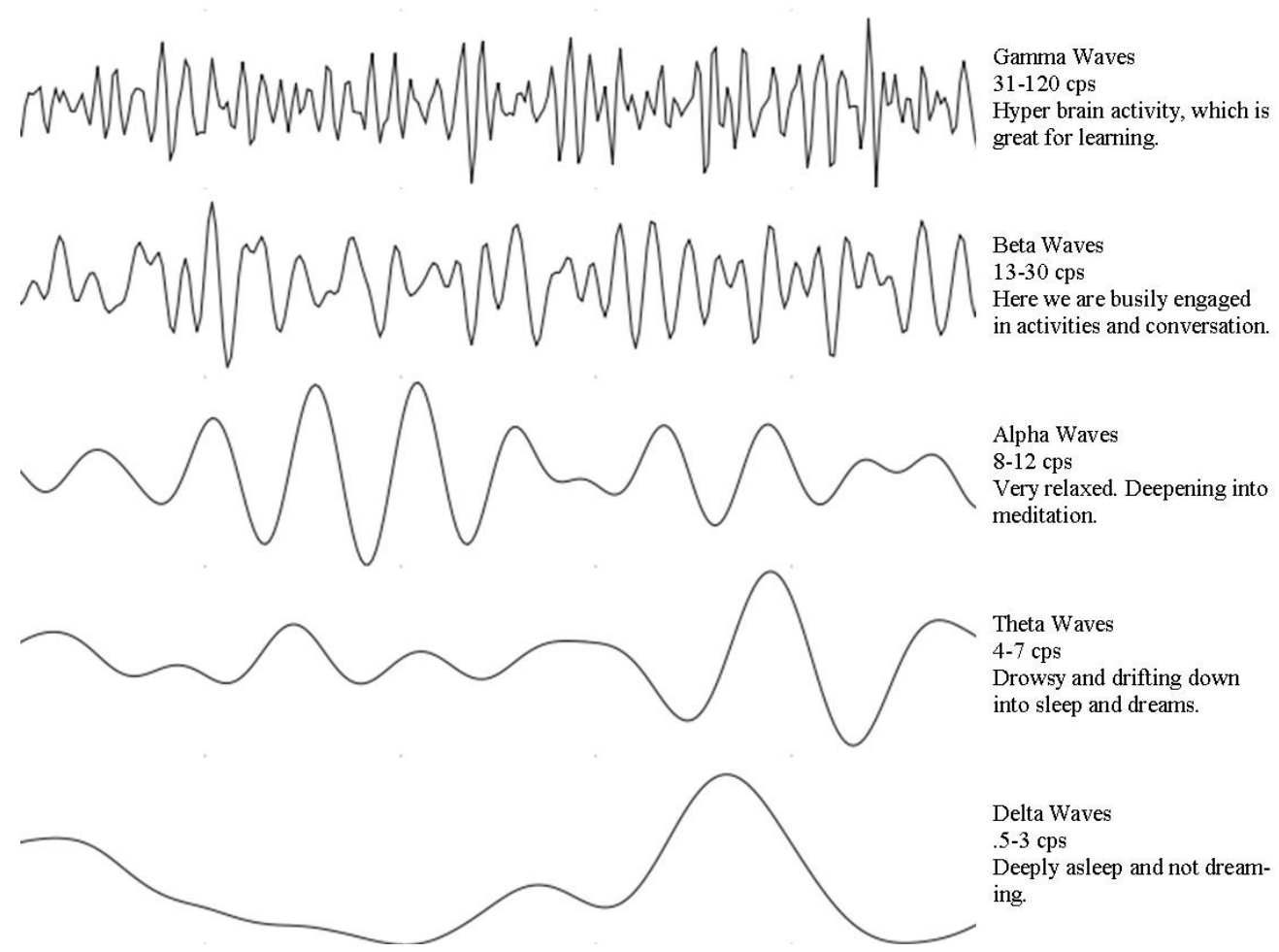

Figure 1. The Brain Waves graph above, from Sparkfun, displays different kinds of brain waves that are experienced during different activities

Most of the current Muse Headband customers use it to enhance and improve the meditation process when doing brain training. By downloading the Muse application associated with Muse Headband, users can create individualized meditation sessions. To create a more relaxing meditation experience for its users, the Muse app developers designed a virtual environment to help visualize users' simultaneous brain activity. Having read the instructions carefully, I set up my own Muse Headband device and connected it with the Muse app. When the session started, the screen display showed a seashore with waves lapping at the sand. When I tried to concentrate, the waves became tranquil; when I was completely relaxed and focused on the session, I could even hear the seagulls from far away. On the other hand, when I was distracted, the waves got stronger and when I intentionally thought about upsetting things, the waves got even louder.

This experience indicates that Muse Headband has the potential to do much more than simply detecting brain waves. In this virtual environment, not only the way the waves act varies, but also the extent to which they act in a certain way changes. This suggests that the Muse Headband not only is able to differentiate different kinds of brain waves, but can also measure the frequency of the waves.

By analyzing the frequency of different types of brain waves, we can estimate a person's emotion. Granted, it is hard to define emotion with specific standards. However, there are some corresponding relations between brain waves and emotions. When in a pleasing and soothing environment, the brain sends out Alpha waves and signs of relaxation and happiness. In contrast, when feeling anxious and angry, the brain sends out Beta signals. Therefore, by identifying different wave types, we could construct a rough idea of the emotions being experienced by patients.

Admittedly, the design and function of Muse Headband is technologically rather basic. In order to precisely measure the frequency of brain waves and thus determine the intensity of the emotions being experienced, additional devices would be necessary. Since the frontal part of the brain is responsible for emotions, increasing the number of sensors around the frontal area would be an effective way to provide more accurate data on the frequency of brain waves. 


\subsection{Prior Work}

Emotion detection is not a new field of study and there are some prior research works on this subject. Although no specific definition for feelings is set, researchers have tried to evaluate emotions using different approaches. An emotion measurement technology company called Affectiva, for instance, has developed a way to recognize emotions based on facial cues and/or physiological responses. As a developer with the Affectiva SDK, I created a program running on Xcode to measure the degree of happiness on a scale from 0.0 to 99.0 based on my facial expression.
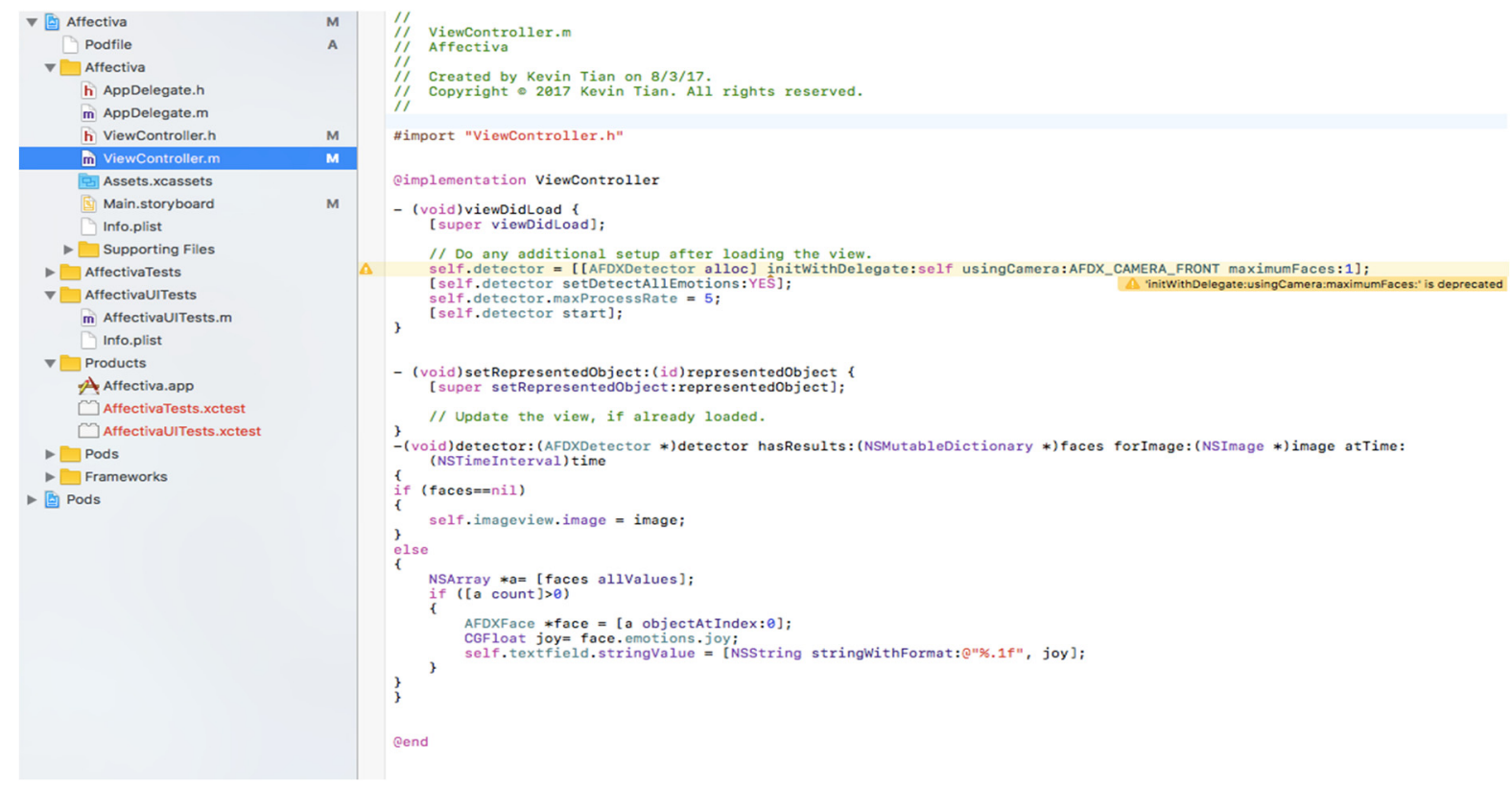

Figure 2. Xcode program for emotion detection

Affectiva is one example among many attempts to build connections between a measurable element and emotion. A group of scholars from the University of California introduced a fascinating product called the Empathy Mirror in a research paper. It is a new interactive device that achieves distant communication. The Empathy Mirror uses a visual interface to capture a user's facial expression and send that expression to his or her partner, thus effectively conveying emotions. Here is a simple example of how the Empathy Mirror works: When Person A is trying to connect to Person B using the Empathy Mirror, B's portrait (on his/her computer screen) displays B's own face with A's emotion. According to the researchers, the Empathy Mirror enhances affective experience, provides a non-verbal form of emotional communication, and improves interaction between people over long distances. It could also help people who lack empathy to develop that ability and may have applications to health care.

Besides attempts at analyzing facial expressions to determine emotions, some researchers have focused on body movements as another approach to measuring emotions. A body movement analysis method introduced by Daniel Bernhardt and Peter Robinson established a system designed to target both the cognitive and physical aspects of an individual's emotional experience. According to the researchers, by using music to mirror the body's physical expressions of emotion, they created a positive feedback effect in an emotionally immersive experience.

Other studies have found that there is a relationship between emotions and brain waves. In an article published by Kate Baggaley on February $1^{\text {st }}, 2017$, she introduces a research project conducted by Niels Birbaumer, a neuroscientist at the Wyss Center for Bio and Neuroengineering in Geneva. Birbaumer and his colleagues are working to communicate with locked-in people by measuring changes in blood flow using a technique called functional near-infrared spectroscopy, which is similar to functional magnetic resonance imaging (fMRI). Essentially, by monitoring the brain's blood flow, the researchers can get an idea of what patients are thinking.

\section{Comparison Experiment}

In order to test the capability of Muse Headband in detecting basic emotions, I designed a comparison experiment between Muse Headband and AffdexMe, an application on Mac based on the Affectiva SDK. My hypothesis was that the Muse Headband would be able to detect the user's fluctuation in emotion, and, to some extent, classify the emotions into different kinds. 


\subsection{Design}

This experiment focused on the detection and comparison of two main emotions: happiness and sadness. The AffdexMe program analyzed my facial muscle movements to determine emotions, while the Muse Headband simultaneously sensed my brain waves. I was looking to see if there would be a correlation in the results. For instance, when I smiled, relaxed, and thought about happy things, I was expecting to see both the AffdexMe indicating happiness and the Muse Headband detecting Alpha brain waves, which would also be a sign of relaxation and calmness. Fundamentally, the main purpose of this experiment was to justify Muse Headband's potential as an emotion detecting device.

\subsection{Material}

1) Muse Headband

2) Macbook Air laptop (with AffdexMe application)

3) iPhone $5 \mathrm{~S}$ (with Muse App associated with the headband)

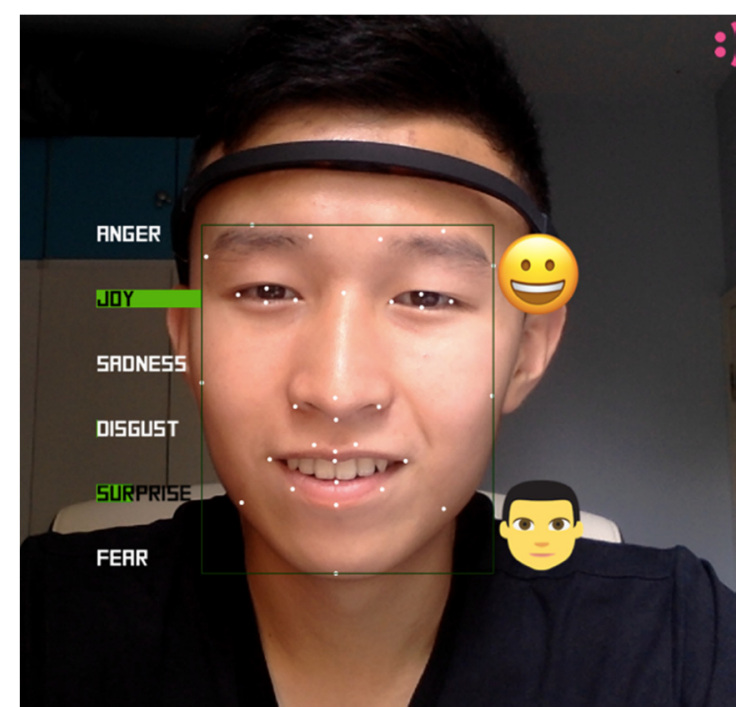

Figure 3. AffdexMe Application feedback

\subsection{Process/Results}

While conducting a 5-minute meditation session on the Muse app, I simultaneously ran the AffdexMe program on the laptop. During the time interval from 0 to $1 \mathrm{~m} 15 \mathrm{~s}$, I recalled happy memories and good news, and slightly smiled to let AffdexMe record my emotion; from $1 \mathrm{~m} 15 \mathrm{~s}$ to $2 \mathrm{~m} 30 \mathrm{~s}$, I made myself upset by thinking about agitating things, and altered my facial expression accordingly. Then I repeated the process. At the end of the experiment, I collected the data from the Muse app.

Not surprisingly, the results justified my hypothesis that the Muse Headband would be able to detect the user's fluctuation in emotion, and, to some extent, classify the emotions into different kinds. Indeed, the data collected by the Muse Headband clearly reflects the different mental states that I intentionally put myself into.

\section{August 12, 2017 - 9:58 AM - 5m}

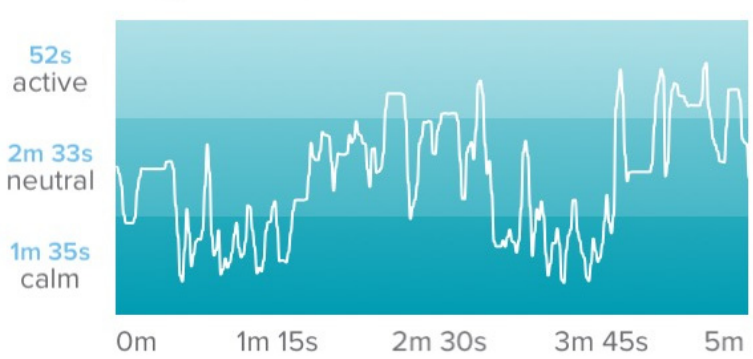

Figure 4. Data collected by the Muse application on iPhone 5S 


\section{Product Designing: Unlock}

Unlock is an in-progress design project based on Muse Headband. The whole program is designed as an approach to help the communication of locked-in people, and consists of both hardware and software.

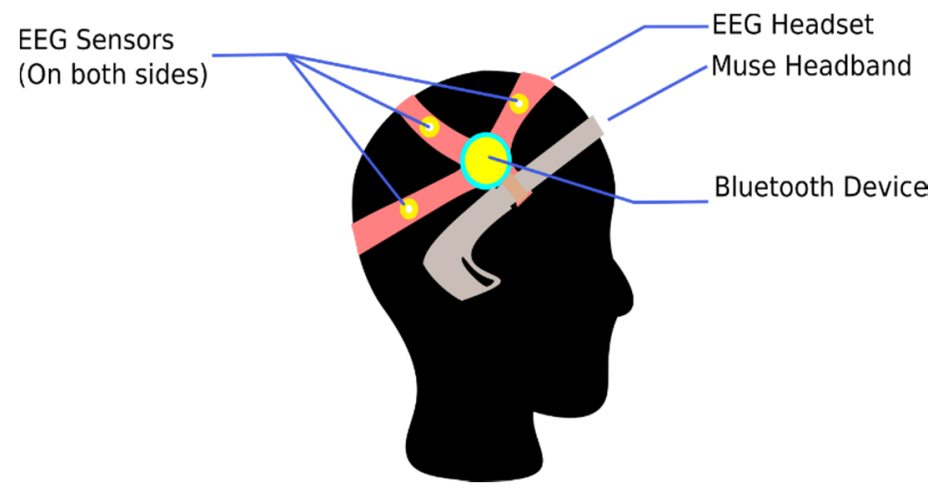

Figure 5. Basic sketch for the conjoint

\subsection{EEG-Muse Headband Headset}

In order to create an approach to detect the emotions of locked-in people by analyzing their brain waves, I come up with a design for a conceptual product based on Muse Headband. I intend to increase the accuracy of the measurement of Muse Headband by increasing the contact area of the sensors. Thus, besides having 3 reference sensors, 2 forehead sensors, and 2 SmartSense Conductive Rubber Ear Sensors from the Muse Headband, the Unlock headset has six additional EEG sensors on both sides of the head. (Figure 5.)

\subsection{Software}

\subsubsection{Simplistic Interface}

Both the headband and the EEGs are connected to a Bluetooth device with a display screen. The output depends on the data collected by the sensing devices. The most simplistic possible way of displaying the output for the data collected and translated can be sketched as shown in Figure 6 .

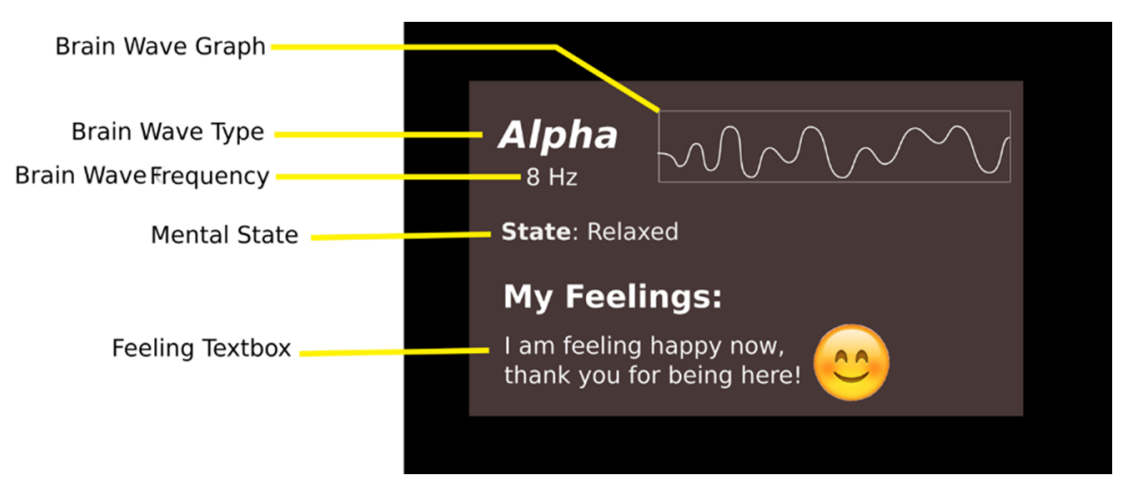

Figure 6. Simplistic interface for the output

As we can see from in Figure 6, there are some basic elements that the display includes: the brain wave graph, the brain wave type, the brain wave frequency, and, most important of all, the emotions of the patient. In this simplistic interface, I have used text boxes and emojis to translate the brain wave analysis into clear notations of feelings. However, despite being relatively easy to achieve and understand, this purely visual presentation of the results might not be the most effective way to truly express a patient's emotions.

\subsection{Virtual Environment and Cartoon Character}

In order to regain a more comprehensive and personalized style of communication between patients and their family members, I need a multisensory program that thoroughly simulates real-life communication. Taking advantage of the 
popularity of smartphones nowadays, this program will take the form of a phone application that is compatible with various systems, such as IOS, Android, etc. The user will be able to pair the sensing device with his/her phone through Bluetooth or an Internet connection, depending on the situation.

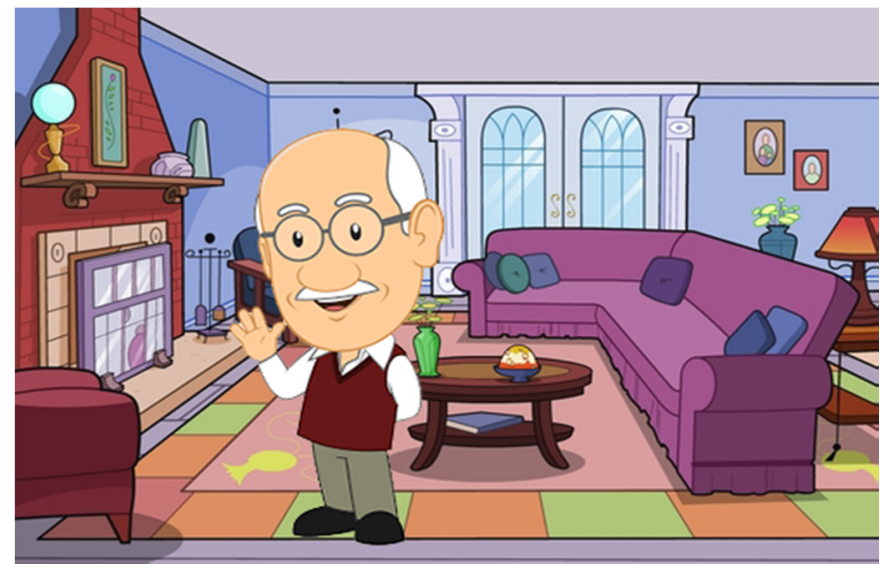

Figure 7. Demonstration of the cartoon designing

The idea for this app is to reflect the emotions of locked-in people using well-designed animations. Here is how it works: The interface of the program will be a virtual environment, a living room for instance. The user will be provided with a few choices for the structure of the living room, and the decoration of the room will be customizable by the users themselves based on real-life experience. Having decided on the environment, a user, presumably the guardian of the patient, will be asked to make a cartoon character based on the appearance of the patient. There will be options for different skin colors, facial features, voices and clothing. When the individualized character and environment are set, the cartoon character will settle down in the room (as seen in the demonstration in Figure 7). Based on the data received by the sensors, the cartoon character will do different things that correspond with the emotions the patient is experiencing. For instance, if a patient is relaxed and amused, which could be inferred by the detection of alpha brain waves, the character in the living room would sit on the sofa with a cup of tea. The frequency of the brain waves matters too-when the amplitude of the alpha waves reaches a certain point, the emotion of the patient can be classified as happy and active. The action of the cartoon character will change along with the fluctuation in emotion: the character will stand up and walk to the window-from which we will hear birds chirping and children laughing; the character will also smile and start whistling. On the other hand, when beta brain waves are detected, which are indicative of uneasiness, the cartoon character will sit on the couch and sigh, or simply walk away into a bedroom in the background. If an abnormal level of anxiety is detected, the character will ask the user to talk to him or her, so that the guardian can know when to check on the patient. Also, when the user taps on the cartoon character, the character will start a dialogue with the user based on the patient's emotion, just like Siri or Contana.

Of course, the setting for the program does not necessarily have to be a living room. It could be a garden, a park, or a beach. The point of the application is to regain what the locked-in people have lost: the joy of communication. With this program, the family members can feel the presence of the patient as a whole person, who has his or her individual characteristics and ways of communicating. The patients will also be happy to see themselves being active and being capable of expressing themselves in such a way. I reckon that if my grandfather had the opportunity to communicate with us in such a way, he would have been very delighted.

\section{Conclusion}

Having ensured the feasibility of using the Muse Headband to collect brain wave data, I now propose the design of a new program for locked-in people's communication: Unlock.

One advantage of the program is that the device used to achieve communication is affordable by an average family. Compared to various other equipment and approaches used by previous researchers, such as functional near-infrared spectroscopy or functional magnetic resonance imaging (which are also not as accurate in terms of getting raw data), the Unlock equipment will cost no more than $\$ 500$, which is a fairly user-friendly price for a brain wave sensing and communication device. 
Moreover, running the program does not require any background knowledge. Instead of being overwhelmed by a myriad of graphs and data, Unlock users would simply receive the information conveyed by animations from the application. The essential focus of this program is to regain a comprehensive and personalized communication between the patient and the family members by simulating real-life communication. Thus, it is essential that both the users and the patients find the program entertaining, easily accessible and considerate.

\section{Acknowlegments}

Thanks to Professor Lorie Loeb for introducing the topic of wearable devices and guiding me through the research process.

\section{Reference}

Developer Kit. (2017). In Muse: the Brain Sensing Headband. Retrieved from www.choosemuse.com/developer-kit/

Affdex for Market Research. (2017). In Affectiva. Retrieved from www.affectiva.com/product/affdex-for-market -research/

Clipart Panda - Free Clipart Images. (2017). Retrieved from www.clipartpanda.com/

Kravitz, S. (2017). Hackers in Residence - Hacking MindWave Mobile. Retrieved from https://learn.sparkfun.com/ tutorials/hackers-in-residence---hacking-mindwave-mobile

\section{Copyrights}

Copyright for this article is retained by the author(s), with first publication rights granted to the journal.

This is an open-access article distributed under the terms and conditions of the Creative Commons Attribution license (http://creativecommons.org/licenses/by/4.0/). 